\title{
Cost analysis and efficacy of recruitment strategies used in a large pragmatic community-based clinical trial targeting low-income seniors: a comparative descriptive analysis
}

\author{
Sravya Kakumanu', Braden J. Manns ${ }^{2,3}$, Sophia Tran², Terry Saunders-Smith², Brenda R. Hemmelgarn², \\ Marcello Tonelli ${ }^{2,3}$, Ross Tsuyuki ${ }^{4}$, Noah Ivers ${ }^{5,6}$, Danielle Southern ${ }^{3}$, Jeff Bakal ${ }^{7}$ and David J. T. Campbell $2,3,8^{*}$
}

\begin{abstract}
Objective: One of the most challenging parts of running clinical trials is recruiting enough participants. Our objective was to determine which recruitment strategies were effective in reaching specific subgroups.

Study design and setting: We assessed the efficacy and costs of the recruitment strategies used in the Assessing Outcomes of Enhanced Chronic Disease Care Through Patient Education and a Value-based Formulary Study (ACCESS) in Alberta, Canada.
\end{abstract}

Results: Twenty percent of the study budget ( $\$ 354,330$ CAD) was spent on recruiting 4013 participants, giving an average cost per enrolled of $\$ 88$ CAD. Pharmacies recruited the most participants $(n=1217)$, at a cost of $\$ 128$ / enrolled. "Paid media" had the highest cost (\$806/enrolled), whereas "word of mouth" and "unpaid media" had the lowest ( $\sim 3 /$ enrolled). Participants enrolled from "seniors outreach" had the lowest baseline quality of life and income, while participants from "word of mouth" had the lowest educational attainment.

Conclusion: The "health care providers" strategies were especially successful — at a moderate cost per enrolled. The "media" strategies were less effective, short lasting, and more costly. No strategy was singularly effective in recruiting our targeted groups, emphasizing the importance of utilizing a variety of strategies to reach recruitment goals.

Trial registration: ClinicalTrials.gov, NCT02579655. Registered on 19 October 2015.

Keywords: Randomized controlled trials, Vulnerable populations, Cost-effective, Recruitment strategies, Seniors, Low enrollment

\section{Introduction}

Randomized controlled trials (RCTs) are widely recognized as the most robust study design for clinical research [1]. One of the greatest challenges in the conduct of clinical trials is participant recruitment [2]. A review

\footnotetext{
* Correspondence: dcampbel@ucalgary.ca

${ }^{2}$ Department of Medicine, Cumming School of Medicine, University of Calgary, TRW 3E33, 3280 Hospital Dr NW, Calgary, AB T2N 4Z6, Canada ${ }^{3}$ Department of Community Health Sciences, Cumming School of Medicine, University of Calgary, Calgary, AB, Canada

Full list of author information is available at the end of the article
}

of 253 terminated trials found that almost $40 \%$ were discontinued prematurely due to difficulties with recruitment [3]. Another study found that up to $50 \%$ of trials had to be extended to enroll a sufficient number of participants, and yet only about $30 \%$ of clinical trials meet their recruitment targets $[4,5]$. As a result of underrecruitment, many studies suffer from a lack of statistical power [6]. This has potential risks: if researchers cannot make their study worthwhile, they waste participants' time and expose them to unnecessary risks without the promise of improvements in clinical care, which is both

(c) The Author(s). 2019 Open Access This article is distributed under the terms of the Creative Commons Attribution 4.0 International License (http://creativecommons.org/licenses/by/4.0/), which permits unrestricted use, distribution, and reproduction in any medium, provided you give appropriate credit to the original author(s) and the source, provide a link to the Creative Commons license, and indicate if changes were made. The Creative Commons Public Domain Dedication waiver (http://creativecommons.org/publicdomain/zero/1.0/) applies to the data made available in this article, unless otherwise stated. 
unproductive and unethical [6, 7]. Recruitment challenges also result in considerable additional costs to extend study recruitment periods [8], which may deter funding institutions from supporting clinical trials [6].

Numerous authors have attempted to explain why recruitment for clinical trials is so difficult. Some have described problems related to study design $[2,9-13]$ and recruitment methodology, such as undesirable trial arms (i.e., control groups) and ineffective recruitment strategies for the target audience $[12,14,15]$. Others have noted challenges specifically inherent to the potential participants $[2,6,11-13]$ and researchers $[2,12,13]$.

Some recent clinical trials have included detailed descriptions about their recruitment process in the published literature. A recent Australian study of older men with diabetes and low testosterone showed successful recruitment through radio and TV advertisements as well as government mail-outs, where all three approaches were relatively cost-effective [16]. Other studies have successfully used health care referrals [16-20], mail-outs [16, 21-23], and community outreach $[18,24]$. However, there are still gaps in our knowledge regarding the effectiveness of various recruitment strategies for different target populations [27], the costs associated with them [28], and their success in reaching specific subgroups, which is particularly lacking in the literature. These knowledge gaps are more prominent for researchers attempting to recruit older participants or socially vulnerable participants, as they are often excluded or underrepresented in the majority of trials [29].

The objective of this paper was to use our experience with the Assessing Outcomes of Enhanced Chronic Disease Care Through Patient Education and a Value-based Formulary Study (ACCESS trial) to address this knowledge gap and to describe the most effective strategies for researchers seeking to enroll low-income seniors into pragmatic clinical trials. The specific objectives included (1) describing the effectiveness and types of patients enlisted by the various recruitment strategies used, (2) detailing the costs associated with each of these strategies, and (3) determining the cost per enrolled participant for each of these strategies.

\section{Methods}

\section{Setting}

The ACCESS trial is a pragmatic clinical trial in Alberta, Canada, which completed recruitment in August 2018 (ClinicalTrials.gov registration NCT02579655). ACCESS is a factorial $(2 \times 2)$ RCT that is evaluating the impact of providing free high-value medications and/or a tailored health education program on patient outcomes and health care costs among seniors at high risk of cardiovascular complications [30]. The free medications intervention involved elimination of patients' $25 \%$ copayments for all medications considered high value in preventing cardiovascular disease (Additional file 1). The personalized education intervention included an online and/or mail-based platform through which participants received tailored health messages. Eligible participants were older people ( $>65$ years) with low incomes $(<\$ 50,000 \mathrm{CAD})$ who had at least one cardiovascular-related chronic disease (coronary artery disease, congestive heart failure, stroke, diabetes, hypertension, chronic kidney disease, hypercholesterolemia). Randomization was conducted in a blinded fashion using permuted block sizes, and group allocation was in a 1:1:1:1 ratio. Participation involved no study visits, and participants were expected to complete three surveys over the course of the 36-month follow-up period. Most outcomes are being tracked using administrative health data. The trial is ongoing, and follow-up is expected to be complete in September 2021.

The ACCESS trial enrolled 4750 participants from November 2015 to August 2018. The present report is an observational study nested within this RCT, based on data from the first 4013 participants enrolled (November 15, 2015 to May 2, 2018).

Interested participants were asked to call a telephone survey unit, regardless of the recruitment strategy by which they initially heard about the ACCESS trial. Patients were instructed to leave a voicemail, and the survey unit would return their call within 48 $\mathrm{h}$ to confirm the participant's interest and complete an initial eligibility assessment. Participants who qualified for the study and enrolled were asked to complete a baseline survey.

\section{Data sources}

Data for this study was collected by self-report from ACCESS trial participants at the time of their initial contact with study staff. Recruitment strategy and basic demographic details were collected during their initial telephone assessment with the survey unit. Missing data was present for individuals who called for information but chose not to be screened for eligibility and for those deemed to be ineligible early in the conversation and who declined further questions (Fig. 1). Detailed demographic information was extracted from the surveys administered to participants at their baseline assessment. In order to enroll in the ACCESS trial, baseline questionnaires (Additional files 2 and 3) must be complete; therefore, no missing data was present for those who enrolled in the trial. Cost data was extracted from a detailed review of study expenses and human resource utilization (Additional file 4). 


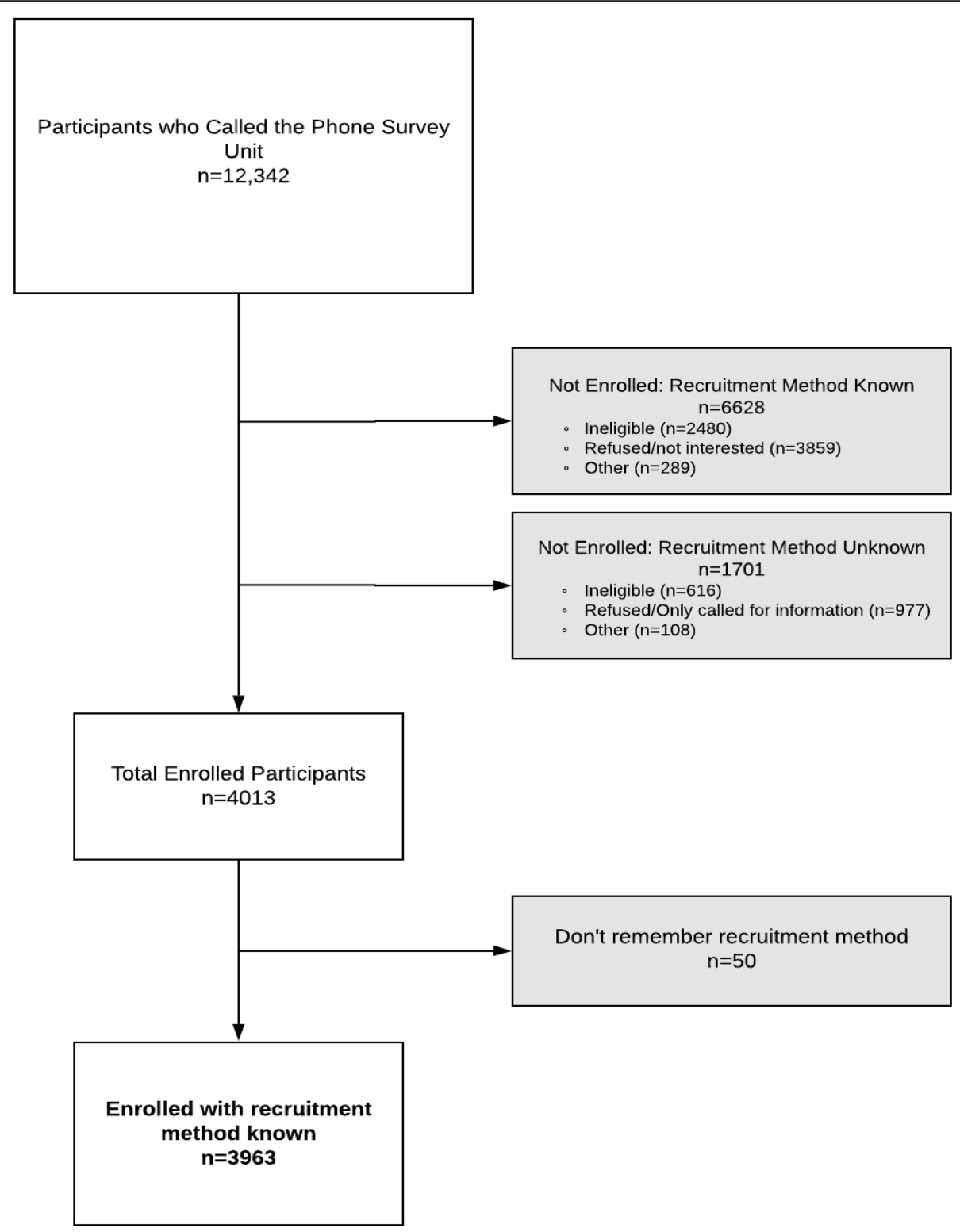

Fig. 1 Flow diagram of potential ACCESS trial participants who made contact with the phone survey unit from November 15, 2015 to May 2, 2018

\section{Variables of interest}

\section{Types of recruitment strategies}

Throughout the 30-month enrollment period, a variety of recruitment strategies were used to identify eligible participants for the ACCESS trial, which we have classified into five overarching strategies and 14 substrategies (Table 1). Participants who called the survey unit were asked an open-ended question to determine how they learned about the study. Responses to this question were allocated to one of the 14 substrategies. Participants who did not remember how they heard about the study were placed in the "Don't Know" category.

\section{Classification of participants}

We classified potential participants who initially contacted the survey unit for eligibility assessment into two major categories: (1) those who eventually enrolled and were randomized, and (2) those who did not enroll for a variety of reasons. This latter group was further classified as either "ineligible", "refused", or "other". Furthermore, all potential participants who provided the recruitment substrategy by which they heard about the study were categorized into the appropriate overarching recruitment strategy.

For all enrolled participants, we extracted demographic information from the eligibility surveys, including urban 
Table 1 Recruitment strategies used in the ACCESS trial

1. Patient contact by health care providers

a. Pharmacies: Several large Canadian pharmacy chains, along with hundreds of independent pharmacies across Alberta, were approached by the ACCESS trial team to be a part of the recruitment process. They were asked to display posters and/or hand out brochures to patients. Pharmacists had considerable autonomy to decide how to recruit patients, with some only having posters displayed, others handing out brochures, and others directly targeting individuals they felt would be appropriate for the study. Regardless, pharmacists did not enroll patients directly but simply provided the number for the study survey unit

b. Health professionals: Throughout the study, we distributed posters and brochures to specialist and family physician offices as well as hospitals throughout Alberta. This category included participants who saw the posters and/or brochures at these locations and participants who were told about the ACCESS trial by medical or allied health professionals (physicians, nurses, and dieticians) other than pharmacists

2. Paper mail

a. Census-based Canada Post mail-out: Brochures were mass mailed to targeted communities identified by Canada Post as having a preponderance of residents who were over the age of 65 and had lower incomes, based on census data. A total of 122,000 brochures were sent out in three separate mailing cycles

b. Coronary angiogram registry: All consenting Albertans who undergo cardiac catheterization for diagnostic or therapeutic purposes are entered into a patient registry called the Alberta Provincial Project for Outcome Assessment in Coronary Heart Disease (APPROACH) [21]. Those who are found to have coronary artery disease are asked to consent to being contacted to hear about future clinical studies. We used the list of those consenting individuals to contact those with demographics suggesting potential eligibility for ACCESS. These individuals were directly mailed a letter about ACCESS. Everyone on the mail-out list was then called by our survey unit to confirm interest and eligibility. A total of 4780 individuals received letters and phone calls over 11 months

c. Targeted mail-out after hospital discharge: Patients who were discharged from an Alberta Health Services (AHS) facility (the single health care provider in Alberta) who met study criteria (based on age and previously known health conditions) were contacted by mail by AHS, inviting them to call the survey recruitment line if they were interested in participating. A total of 50,042 letters were sent over 11 months

3. Media

a. Paid radio advertisements: Three local radio stations in Calgary and nearby areas were selected on the basis of having a large audience with the correct target demographic to play 30-s commercials up to 42 times a week for 2 weeks. The radio commercial included instructions on how to enroll and a brief summary of the study, highlighting that eligible individuals would have a $50 \%$ chance to receive free medications

b. Facebook: A paid Facebook ad was designed to recruit participants (disseminated to Alberta seniors and a separate Facebook ad to younger individuals - noting the potential relevance to their parents and grandparents) and was displayed on the pages of targeted groups for a few months

c. Hospital programming channel: A professionally produced 90-s TV commercial aired on televisions in select physicians' offices, community laboratories, and some AHS facilities throughout Alberta for 6 months in 2016/2017

d. Transit advertisements: Advertisements were placed at transit stops and stations as well as inside the trains and buses in Calgary,

Edmonton, Medicine Hat, and Lethbridge for 11 months
Table 1 Recruitment strategies used in the ACCESS trial (Continued)

e. Paid print media: Included anyone recruited from paid print advertisements. This included advertisements placed in general and senior-specific newspapers and newsletters throughout the province

f. Unpaid media: Included free social media advertising from personal, university, and charitable organizational accounts on Twitter and Facebook. Press releases were sent from the University of Calgary in March 2016 and from AHS in September 2016. A variety of coverage arose from this - predominantly radio stories and interviews. Two news stories featuring the ACCESS trial were broadcast on local TV stations during the evening local news. Various other free stories and articles in papers and newsletters across Alberta arose from a variety of other contacts. Anyone who claimed to see any print, online, or aired media within 3 weeks of the release dates was categorized under "unpaid media"

4. Seniors outreach

a. Seniors' homes: The ACCESS team traveled to and contacted various seniors' homes and apartments to give presentations and drop off brochures and/or posters. Many rural locations were contacted by phone, and if managers were interested in recruiting, posters and brochures were sent directly to the seniors' homes. Some seniors' homes posted the materials in common areas, while others put brochures directly in mailboxes or under residents' doors

b. Seniors aid resources: Seniors aid resources included social workers, help centers, food banks, and health care or social care service coordinators. Advertising to these places/people consisted of word of mouth and the distribution of brochures to institutions and providers. Any presentations or booths set up by the ACCESS team at events, fairs, or centers were also included in this category

5. Word of mouth: This strategy was established as the study became more well known. Many study participants told their family members and/or friends about the study and gave them the enrollment phone number. As this strategy became more successful, we further encouraged it by periodically sending enrolled participants recruitment brochures to distribute to those who might be eligible and interested

versus rural residence, first language, income category, educational attainment, age category, prescribed medications, and quality of life. This was done to determine the effectiveness of the various recruitment strategies at targeting specific populations.

\section{Recruitment costs}

We also estimated detailed costs associated with each of the 14 strategies. The total costs included both supplies and services costs, as well as human resources costs. Supplies and services costs included expenses for materials (brochures and posters), travel, advertising fees, postage fees, and consulting fees. All promotional content was developed by the study team and was approved by the local ethics boards before distribution. Focus groups conducted later in the study provided some feedback on the promotional content [25]. Human resources costs included the time required by the ACCESS team (research assistants and research coordinator) to start, organize, and sustain the strategy, and, in the case where the participants were actively called by the survey unit (coronary angiogram registry), the time required by the survey unit to call participants (Additional file 4). The human resources costs 
did not include the time of study investigators or coinvestigators who oversaw the recruitment effort.

\section{Data analysis \\ Efficacy of paper mail strategies}

The mail-based strategies provided a unique opportunity to calculate a denominator (i.e., knowing how many people were initially contacted by each strategy). Therefore, we determined how successful each of these strategies were at getting recipients to actually call and enroll in the study. We calculated two measures of effectiveness for each strategy: (1) the proportion of initial contacts who received mail who ended up enrolling in the study (proportion of initial contacts), and (2) the proportion of those who actually called who completed enrollment (proportion of callers). The first percentage was calculated by dividing the number of enrolled by the total number of individuals who were sent mail, and the second percentage was calculated by dividing the number of enrolled by the number of calls received by the survey unit.

\section{Demographic analysis}

The sociodemographic characteristics of the participants enrolled were compared across recruitment strategies with the goal of determining whether the strategies differed in their ability to reach different types of patients. In particular, we were interested in between-strategy differences in effectiveness of recruiting individuals from typically under-represented and difficult-to-recruit subgroups.

For each recruitment strategy, the proportion of its enrolled participants from each demographic category was calculated, as well as corresponding 95\% confidence intervals (CIs) using the binomial distribution. A quality of life measurement was also included as a demographic characteristic of the enrolled participants. Quality of life was calculated using the EuroQol five-dimension, five-level (EQ$5 \mathrm{D}-5 \mathrm{~L}$ ) scoring system, a measure of overall well-being that gives a score from 0 to 1 , with 1 representing perfect health.

\section{Timeline analysis}

We were interested in whether media recruitment strategies resulted in sustained interest in the study. We therefore plotted media recruitment by the week of study and superimposed the various media recruitment strategies.

\section{Cost analysis}

Finally, by using the estimated costs incurred by each strategy, we were able to calculate a strategy-specific cost per enrolled participant. This was calculated by dividing the total cost of the strategy by the number of participants enrolled by the strategy.

\section{Results}

\section{Study cohort}

From November 2015 until May 2018 a total of 12,342 people called the survey unit (Fig. 1). Of these potential participants, 4013 were randomized, of whom 50 were unable to recall how they heard about the study. Of the 8329 potential participants who did not enroll, 1701 did not specify how they heard about the study either because they were disqualified too early during the phone survey to answer the question or they had just called for information, not intending to enroll.

\section{Analysis of recruitment strategies Demographic analysis}

Given that ACCESS was attempting to enroll individuals who were less likely to be on optimal medical therapies, the demographics that were most important to target in this study were older seniors with lower incomes, those not on indicated medications (statins, angiotensinconverting enzyme $[\mathrm{ACE}]$ inhibitors/angiotensin receptor blockers [ARBs]) at baseline, those with lower levels of educational attainment, and those with a lower quality of life. Nearly $1 / 3$ (29\%) of participants enrolled through the coronary angiogram registry were above the age of 80 , making it the strategy with the highest proportion of older seniors (Table 2). Participants from seniors outreach were the most likely to be in the lowest income bracket $(74 \%$ had an income $<\$ 30,000$ CAD). Pharmacies recruited the most people with less than a high school education $(n=$ 379), but word of mouth, although recruiting fewer than half as many participants, still a slightly higher proportion of its enrolled participants with less than a high school education at $35 \%(n=166)$. Only $8 \%$ of all participants were not on either class of indicated medication; health professionals and seniors outreach had the highest proportion $(12 \%)$ of their enrolled participants meeting this criteria. Finally, the utility scores showed that participants from seniors outreach had the lowest quality of life with an average EQ-5D score of 0.617 , while participants recruited from the coronary angiogram registry had the highest quality of life with an average score of 0.686 .

\section{Recruitment success}

The largest proportion of our 4013 participants was enrolled from health care providers $(n=1527 ; 38 \%)$, with the largest group coming from pharmacies $(n=1217$; 30\%) (Table 2). The mail-based strategies recruited 1358 participants (34\% of the total), while paid media only recruited 85 individuals ( $2 \%$ of all participants). Each of the individual strategies under paid media recruited fewer participants than any other strategy used in the study (Table 3).

Despite this broad variation in success in prompting individuals to call the survey unit, the recruitment strategies 


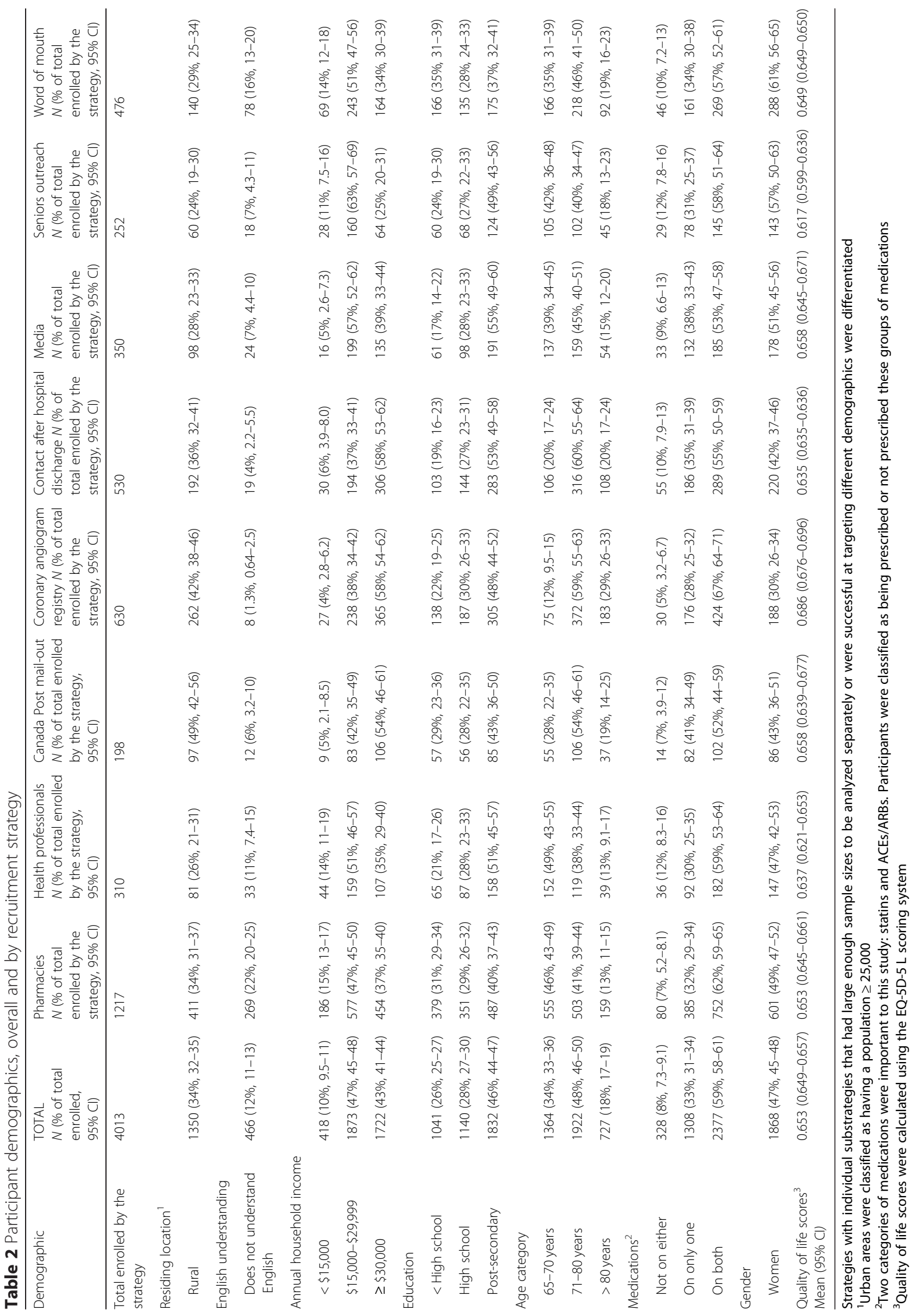


Table 3 Summary of participants enrolled and cost breakdown, by recruitment strategy

\begin{tabular}{|c|c|c|c|c|c|}
\hline Recruitment strategy & $\begin{array}{l}\text { Number of enrolled } N \text { (\% of total } \\
\text { enrolled, } 95 \% \mathrm{Cl} \text { ) }\end{array}$ & $\begin{array}{l}\text { Supplies and services } \\
\text { cost }(\$ C A D)\end{array}$ & $\begin{array}{l}\text { Human resources } \\
\text { cost }(\$ C A D)\end{array}$ & $\begin{array}{l}\text { Total cost } \\
(\$ C A D)\end{array}$ & $\begin{array}{l}\text { Cost per enrolled } \\
\$ C A D / \text { participant }\end{array}$ \\
\hline Health care & $1527(38 \%, 37-40)$ & 66,690 & 91,910 & 158,600 & 104 \\
\hline Pharmacies & $1217(30 \%, 29-32)$ & 63,500 & $91,480^{a}$ & 154,980 & 128 \\
\hline Health professionals & $310(7.7 \%, 6.9-8.6)$ & 3190 & $430^{a}$ & 3620 & 12 \\
\hline Paper mail & $1358(34 \%, 32-35)$ & 90,770 & 15,370 & 106,140 & 78 \\
\hline $\begin{array}{l}\text { Canada Post mail-out } \\
(n=122,000)\end{array}$ & $198(4.9 \%, 4.3-5.7)$ & 39,400 & $300^{a}$ & 39,700 & 201 \\
\hline $\begin{array}{l}\text { Coronary angiogram } \\
\text { registry }(n=4780)\end{array}$ & $630(16 \%, 15-17)$ & 4780 & $12,670^{\mathrm{b}}$ & 17,450 & 28 \\
\hline $\begin{array}{l}\text { Contact after hospital } \\
\text { discharge }(n=50,042)\end{array}$ & $530(13 \%, 12-14)$ & 46,590 & $2400^{b}$ & 48,990 & 92 \\
\hline Media & $350(8.7 \%, 7.9-9.6)$ & 66,610 & 2940 & 69,550 & 199 \\
\hline Paid media & $85(2.1 \%, 1.7-2.6)$ & 66,610 & 2040 & 68,650 & 808 \\
\hline Paid radio & $13(0.32 \%, 0.19-0.55)$ & 11,850 & 120 & 11,970 & 921 \\
\hline Facebook & $2(0.050 \%, 0.014-0.018)$ & 10,200 & $300^{a}$ & 10,500 & 5250 \\
\hline $\begin{array}{l}\text { Hospital programming } \\
\text { channel }\end{array}$ & $8(0.20 \%, 0.10-0.39)$ & 10,220 & $600^{a}$ & 10,820 & 1353 \\
\hline Transit advertising & $26(0.65 \%, 0.44-0.95)$ & 23,040 & 120 & 23,160 & 891 \\
\hline Print media & $36(0.90 \%, 0.65-1.2)$ & 11,300 & $900^{a}$ & 12,200 & 339 \\
\hline Unpaid media & $265(6.6 \%, 5.9-7.4)$ & 0 & $900^{\mathrm{a}}$ & 900 & 3 \\
\hline Seniors outreach & $252(6.3 \%, 5.6-7.1)$ & 12,380 & 4260 & 16,640 & 66 \\
\hline Senior's homes/apartments & $74(1.8 \%, 1.5-2.3)$ & 5690 & $4100^{a}$ & 9790 & 132 \\
\hline Senior's aid resources & $178(4.4 \%, 3.9-5.2)$ & 6690 & $160^{a}$ & 6850 & 38 \\
\hline Word of mouth & $476(12 \%, 11-13)$ & 2200 & 1200 & 3400 & 7 \\
\hline TOTAL & 4013 & 238,650 & 115,680 & 354,330 & 88 \\
\hline
\end{tabular}

${ }^{a}$ Cost calculated using research assistant salary at approximately $\$ 30 \mathrm{CAD} / \mathrm{h}$

${ }^{b}$ Cost calculated using research coordinator salary at approximately $\$ 60 C A D / h$

generally seemed to enroll a similar proportion of initial callers $(\sim 60 \%)$ (Fig. 2). However, this value was only calculated using the potential participants who provided their recruitment strategy; 1701 callers did not provide this information (Fig. 1.) The exception to this generalization was the mail strategies, where there was considerably more variation (Table 4).

\section{Mail strategies}

Of the 176,822 people who were initially contacted by mail, only $3.61 \%(n=6384)$ contacted the telephone survey unit (Table 4). Furthermore, only $21.3 \%(n=1358)$ of these callers were eligible and consented to enrollment, which meant only $0.8 \%(1358 / 176,822)$ of all people contacted by mail actually enrolled in the study. Regarding the specific mail strategies: the coronary angiogram registry had $100 \%(n=4780)$ of contacts complete the phone survey (due to the fact that we actively called them). However, with only 630 of the potential participants enrolling, it successfully enrolled the lowest percentage of those who were spoken to on the phone (13.2\%) compared to the other mail-based strategies. On the other hand, the Canada Post mail-out had the lowest proportion of contacts who called in $(0.25 \%, n=300)$, but those who called in were much more likely to enroll, with a proportion similar to the non-mail strategies $(66.0 \%, n=$ 198). The contact after hospital discharge strategy only had $2.61 \%(n=1304)$ of its initial contacts call the phone survey unit, and with $40.6 \%$ enrolling $(n=530)$, it had a smaller proportion of its initially contacted participants enroll, compared to other strategies.

\section{Recruitment timeline}

Various recruitment strategies caused discrete and noticeable increases in recruitment. Particularly, the press releases, and some of the unpaid media efforts, resulted in a measurable uptick in recruitment. Notably, none of the strategies seemed to cause any degree of sustained recruitment, as each increase only lasted for 1-2 weeks. The first press release was considerably more impactful than the second. Many of the smaller spikes in the later weeks of the study did not coincide with our paid media strategies and could only be attributed to unpaid media, or word of mouth (Fig. 3). 


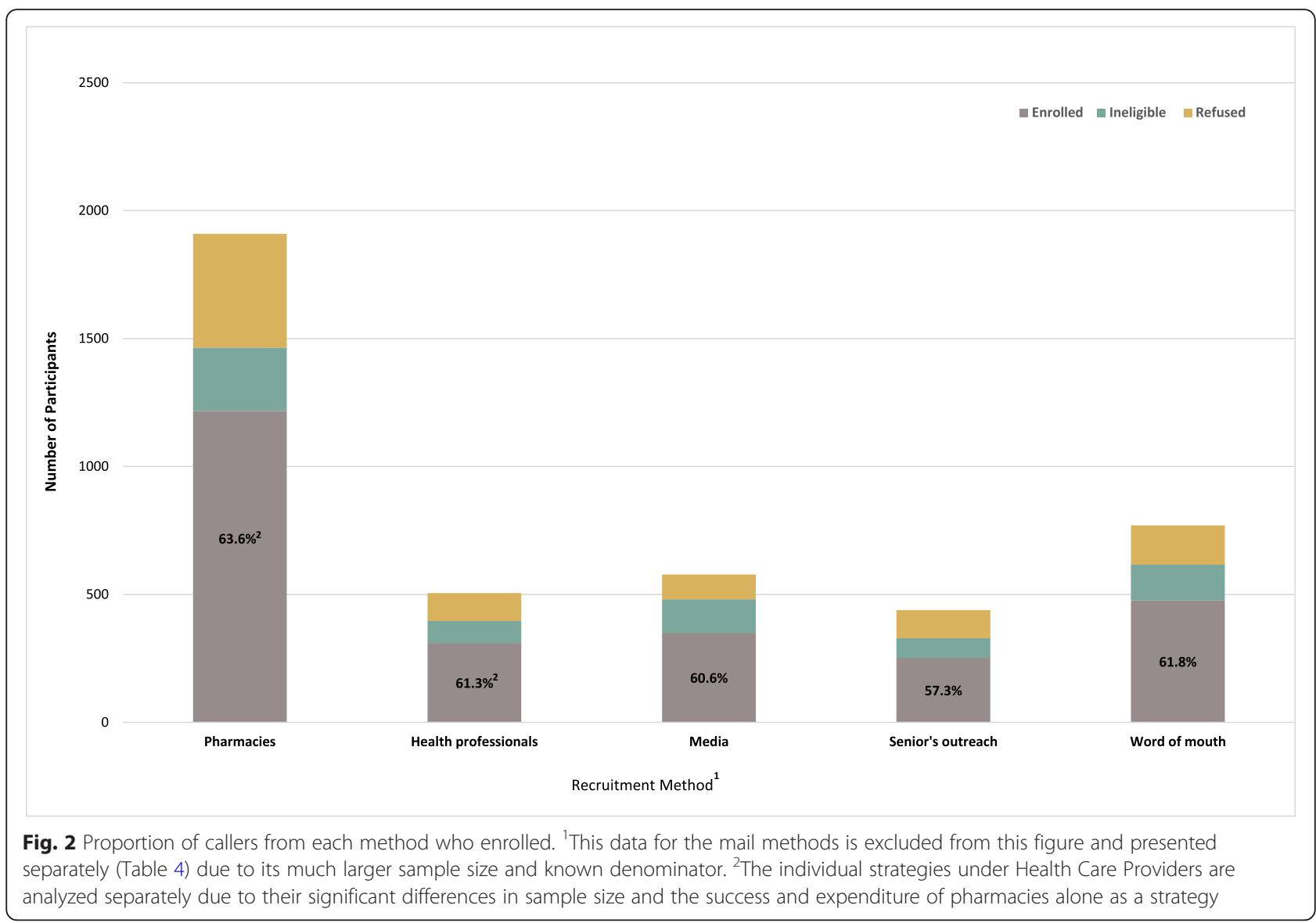

\section{Costs of recruitment strategies}

The total cost of recruitment was $\$ 354,330$ CAD (Table 3). Nearly half of this sum was spent on health care provider recruitment $(\$ 158,600)$, which was the most successful strategy. As per the recruitment plan, most of the human resources were spent on pharmacies rather than other health professionals because they were located in the community, they tended to be more inclined to become involved with research, and people were likely to contact their pharmacist often. The study team spent most of their time informing pharmacies about the ACCESS trial and continuously sent out posters and pamphlets to enable them to advertise for us. Involvement of other health providers in recruitment was done on an ad hoc basis, when the team was contacted by providers looking to get involved, and therefore required much less employee time. The least costly recruitment strategies were word of mouth (\$3400 CAD) and unpaid media (\$900 CAD), which both recruited small numbers of participants. The cost per enrolled participant varied greatly between and within the overarching strategies, with an overall average cost of recruitment of $\$ 88$ CAD per enrolled participant (Table 3). Of the recruitment strategies, paid media had the highest cost per enrolled at $\$ 808 \mathrm{CAD} /$ participant, which included paid radio

Table 4 Effectiveness of mail strategies

\begin{tabular}{|c|c|c|c|c|c|}
\hline \multirow[t]{2}{*}{ Mail-out strategy } & \multirow{2}{*}{$\begin{array}{l}\text { Initial } \\
\text { Contacts } \\
\text { (total } \\
\text { mailed) }\end{array}$} & \multirow{2}{*}{$\begin{array}{l}\text { Number of } \\
\text { calls into } \\
\text { VOXCO } \\
N \text { (\% callers } \\
\text { out of total } \\
\text { mailed) }\end{array}$} & \multicolumn{3}{|c|}{ Contacts who ended up enrolling in the study } \\
\hline & & & $N$ & $\%$ enrolled out of total mailed & $\%$ enrolled from total number of calls \\
\hline All mail strategies & 176,822 & $6384(3.61 \%)$ & 1358 & $0.768 \%$ & $21.3 \%$ \\
\hline Canada Post mail-out & 122,000 & $300(0.246 \%)$ & 198 & $0.162 \%$ & $66.0 \%$ \\
\hline Coronary angiogram registry & 4780 & $4780(100 \%)^{a}$ & 630 & $13.2 \%$ & $13.2 \%$ \\
\hline Contact after hospital discharge & 50,042 & 1304 (2.61\%) & 530 & $1.06 \%$ & $40.6 \%$ \\
\hline
\end{tabular}

${ }^{a}$ This strategy was unique in that we actively called all contacts, rather than simply providing them with the survey unit phone number 
Media Recruitment Methods

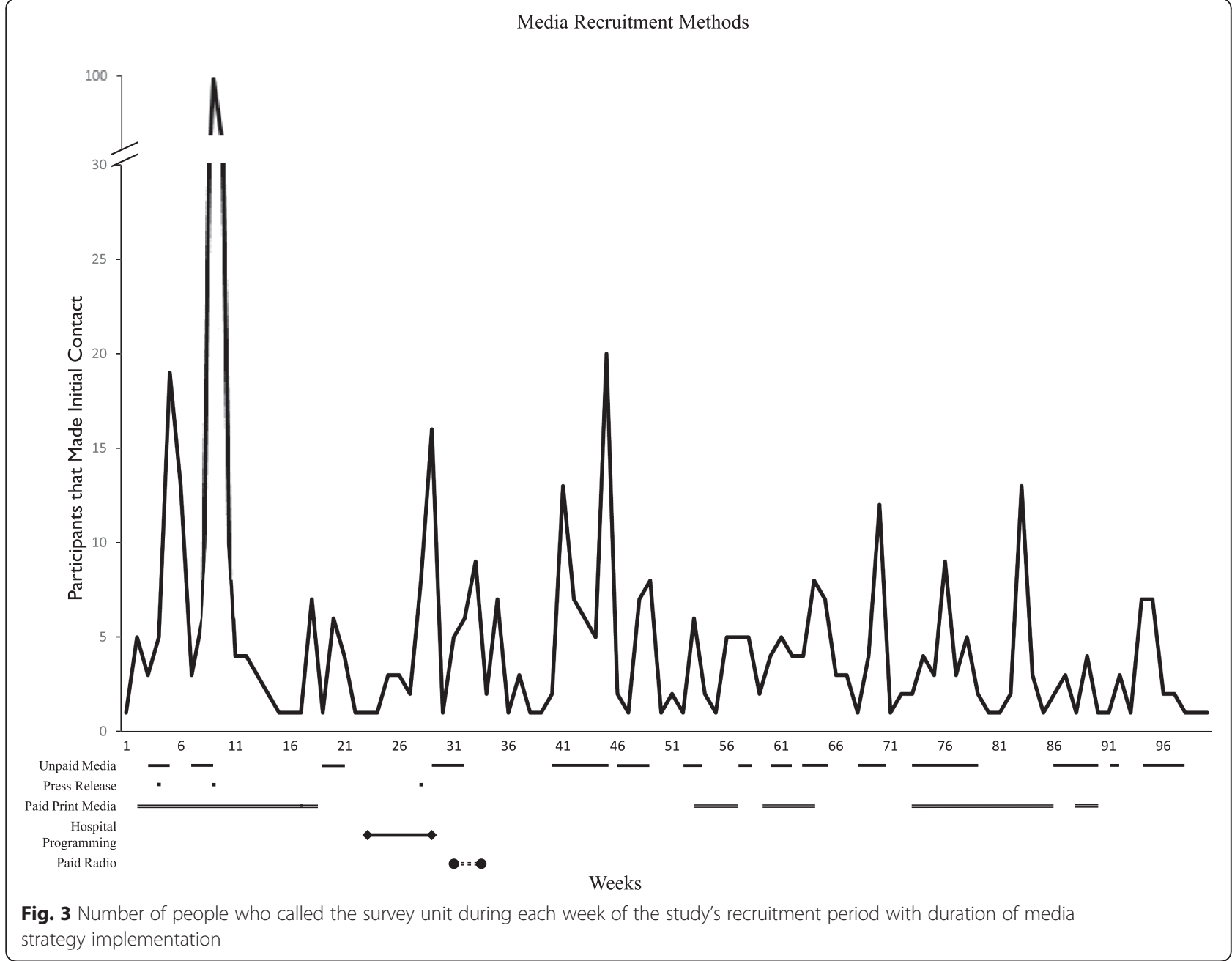

(\$921 CAD/participant), Facebook (\$5250 CAD/participant), hospital programming channel (\$1353 CAD/participant), transit advertising ( $\$ 891 \mathrm{CAD} /$ participant), and print media (\$339 CAD/participant). This is in contrast to unpaid media, which had the lowest cost per enrolled of all 14 substrategies at $\$ 3 \mathrm{CAD} /$ participant. Despite the high total cost of the pharmacy substrategy, its cost per enrolled was only slightly above the average at $\$ 128 \mathrm{CAD} /$ participant.

\section{Discussion}

Recruitment for ACCESS was time-consuming and costly, but ultimately successful. We used 14 substrategies to recruit the first 4013 participants into the study, at a cost of $\$ 354,330 \mathrm{CAD}$, which was approximately $20 \%$ of the overall study operating expenditures during this period. Despite eventual success, there was a lack of adequate planning and budgeting at the beginning of the study to successfully reach the target number of participants. Initial planning set out 12 months for recruitment, with only $\$ 20,000$ CAD set aside as a dedicated recruitment budget to contact pharmacies, which was to be the sole recruitment strategy. As recruitment continued, the timeline had to be extended and considerably more resources had to be put towards recruitment-all of the other strategies developed once it became clear that the initial plans for recruitment were going to be inadequate. It is important for researchers to understand how much of the study budget may need to be spent on recruitment, and to plan their funding requests accordingly. No single strategy appeared to succeed in recruiting typically under-represented groups; rather, the strategies differed in their ability to recruit various types of people. This suggests that the use of multiple and varied recruitment strategies is important to increase the likelihood of achieving sociodemographic diversity $[2,12,26]$. The average cost per enrolled participant was \$88 CAD; however, the costs involved with each strategy used in the ACCESS trial varied considerably. It is important to recognize how the availability of resources can restrict recruitment efforts, thus influencing what is seen as a successful recruitment strategy. As found in the ACCESS trial, each strategy differs in its cost, ability to 
recruit participants (including specific types), and timeliness.

Under-recruitment and the high costs of recruiting for clinical trials have been well documented in the literature. Many authors have provided insight on how to change study designs to help researchers reach recruitment goals within their desired budget and time frame [9]. Building upon this work, there were knowledge gaps our study was able to address, most importantly, how to recruit populations which are typically underrepresented in clinical research. Most studies that have analyzed the factors that impact recruitment (including the studies included in Treweek's meta-analysis [9]) were enrolling younger and healthier participants and not actively targeting low-income seniors with chronic diseases (at high risk of hospitalization). Another important difference is that our study concentrated on understanding the effectiveness of specific recruitment strategies and their associated costs, whereas Treweek's work had examined other aspects of the study design that can affect recruitment success (i.e., the consent process, the initial information given to potential participants, incentives, trial conduct, etc.) [9]. The study published by Bracken et al. [16] provides a cost analysis and detailed explanations of many commonly used and successful strategies such as radio and TV advertisements, health care referrals, mailouts, and community outreach which were also used in the ACCESS trial; however, our study targeted a different participant base and also provides a demographic analysis of the subtypes of participants recruited by each strategy. The availability of both of these studies provides future researchers with greater insight on the types of participants to expect from various recruitment strategies, depending on their target demographic. By thoroughly analyzing each individual strategy, our paper provides researchers different perspectives on what could be considered a successful recruitment strategy, allowing them to determine which might be most suitable for their studies.

A common barrier to the recruitment of older participants is lack of trust in the researchers and recruiters [11-13, 26]. This implies that recruitment may be more successful if participants hear about studies from trusted individuals, such as their physicians or pharmacists [2, 27], and it might explain why health care providers enrolled the most participants in our study. Similarly, personal referrals have been shown to be quite successful at persuading people to enroll $[2,11-13$, $26,28,32,33]$; which we also noted with the success of the "word of mouth" strategy. Mail-based recruitment strategies can be challenging to implement. Suggestions for improving the success of mail-based recruitment strategies include personalizing the letters as much as possible using a prespecified list that is more likely to include only individuals who are most likely to be eligible and interested [26]. These factors might explain why two of our mail strategies (coronary angiogram registry and contact after hospital discharge) were considerably more successful than the more generic Canada Post mail-out $[2,11,26,34]$. However, it is important to note that even though the coronary angiogram registry had a high human resources cost, the active approach of calling participants directly made this strategy much more successful than the other mail strategies, which were more passive. Community-based approaches and cultural adaptation are known to enhance recruitment efforts [11] — which we saw in the moderate success of our outreach strategies. In terms of the ratio of men and women enrolled, each strategy had a relatively equal number of men and women recruited with the exception "word of mouth" and the coronary angiogram registry. "Word of mouth" and "seniors outreach" recruited a higher number of females, potentially because females have been observed to be slightly more social than males [35]. The coronary angiogram registry recruited a higher proportion of males, most likely due to the fact that on average males have cardiovascular events earlier than females [36], making them more likely to be on the coronary angiogram registry.

As we observed, social media is typically thought to be more effective at reaching younger populations than low-income seniors who may not be as technologically inclined [13, 15, 27]. Previous studies have found the total costs of media recruitment to be relatively low [32], which was true for ACCESS as well. However, we had very few participants recruited by paid media, making its cost per enrolled participant quite high. In contrast to paid media, we found that unpaid media (such as coverage stemming from press releases) had the lowest cost per enrolled out of all strategies. This emphasizes the importance of using free advertising and building trust with local communities to help with the promotion of study recruitment [14]. Media coverage seemed to generate immediate interest in the study that was not sustained over time. It is quite clear that the first major press release in March 2016, which garnered much interest in unpaid print and online media, generated a clear spike in initial contacts.

Our analysis has some limitations that merit discussion. First, due to the contextual nature of this work, the findings of this study may only be generalizable to researchers studying a similar population (low-income seniors with chronic diseases) who are offering the possibility of a tangible benefit to study participants (i.e., free medications). Furthermore, the specific costs, and particularly those related to human resources, are highly contextual. Other researchers should anticipate that the exact costs of specific strategies will vary within their own locations, but the relative costs should be generalizable to all settings of similar studies. Second, we do not have detailed 
information on why individuals declined to participate, which could be useful in the future to modify recruitment approaches. Third, we were unable to account for the impact that media promotion may have had on other recruitment strategies. It is possible that a number of health care providers, senior outreach workers, and friends and family members who referred participants to our study initially heard about it from media sources. Similarly, participants might have heard about the study from multiple sources, but only called the study after the most recent contact. Therefore, the reach of some strategies may have been underestimated. Finally, this quantitative analysis does not offer any insights into why some strategies were more effective or how success was achieved. This would necessitate a qualitative inquiry, which may be informative to researchers - such an analysis has been conducted by our team separately [25].

\section{Conclusion}

In summary, we found that different recruitment strategies were better at targeting certain demographic groups and varied in their overall effectiveness. Overall, recruitment through personal referrals from health care providers was the most expensive but enrolled the most participants. However, strategies such as unpaid media and word of mouth-while enrolling fewer participantswere associated with the lowest cost per enrolled, highlighting the importance of community outreach methods in recruiting populations that are typically under represented in clinical trials. The experience of the ACCESS trial emphasizes the importance of understanding the target demographic of a clinical study and determining the most appropriate strategies to effectively recruit those desired individuals.

\section{Additional files}

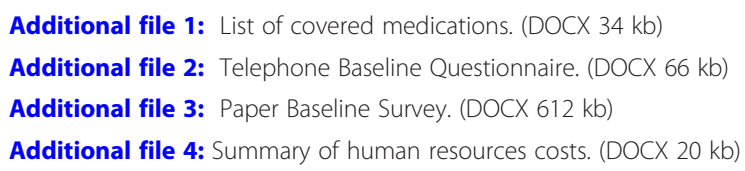

\section{Abbreviations}

ACCESS: Assessing Outcomes of Enhanced Chronic Disease Care Through Patient Education and a Value-based Formulary Study; ACE: Angiotensinconverting enzyme; ARB: Angiotensin receptor blocker; RCT: Randomized controlled trial

\section{Acknowledgements}

We would like to acknowledge the help of ACCESS staff members Patricia Wiebe, Alexa Desjarlais, Charlotte Martin, and Jane Fletcher for their efforts to ensure successful recruitment. We are grateful to all pharmacists, health care providers, and media organizations who helped promote the ACCESS trial.

\section{Authors' contributions}

DJTC, BJM, RT, BRH, and MT sit on the steering committee for the ACCESS trial and therefore led the recruitment efforts described in this paper. $\mathrm{NI}, J \mathrm{~B}$, and DS were advisers to the steering committee for recruitment efforts. SK, BJM, DJTC, and TSS contributed to the conceptualization of the study. SK, ST, and TSS conducted the data collection and data analysis under the supervision of BJM and DJTC. SK wrote the initial draft of the manuscript. All authors contributed to the interpretation of the study data and critically revised the manuscript draft and approved the final manuscript.

\section{Funding}

The ACCESS trial was funded by Alberta Innovates - Health Solutions (Collaborative Research and Innovation Opportunities [CRIO] Program), the University of Calgary Clinical Research Fund, and a Canadian Institutes of Health Research Foundation Grant (to B.J. Manns). Dr. Campbell's salary was supported by a Clinician Fellowship from Alberta Innovates. Ms Kakumanu's salary was supported by an undergraduate summer studentship from the University of Calgary's Program for Undergraduate Research Experience (PURE).

\section{Availability of data and materials}

The datasets used and/or analyzed during the current study may be made available from the corresponding author on reasonable request.

Ethics approval and consent to participate

The ethics ID for the ACCESS trial is REB13-1241. No ethical approval was required specifically for this study.

Consent for publication

Not applicable.

\section{Competing interests}

Dr. Tsuyuki has received investigator-initiated grants from Merck Canada, AstraZeneca Canada, and Sanofi Canada. He has given paid presentations for Merck Canada and Sanofi Canada.

\section{Author details}

${ }^{1}$ Department of Biological Sciences, Faculty of Science, University of Calgary, Calgary, AB, Canada. ${ }^{2}$ Department of Medicine, Cumming School of Medicine, University of Calgary, TRW 3E33, 3280 Hospital Dr NW, Calgary, AB T2N 4Z6, Canada. ${ }^{3}$ Department of Community Health Sciences, Cumming School of Medicine, University of Calgary, Calgary, AB, Canada. ${ }^{4}$ Department of Pharmacology, Faculty of Medicine and Dentistry, University of Alberta, Edmonton, AB, Canada. ${ }^{5}$ Department of Family \& Community Medicine, Faculty of Medicine, University of Toronto, Toronto, ON, Canada.

${ }^{6}$ Department of Family \& Community Medicine, Women's College Hospital, Toronto, ON, Canada. ${ }^{7}$ Department of Medicine, Faculty of Medicine and Dentistry, University of Alberta, Edmonton, AB, Canada. ${ }^{8}$ Department of Cardiac Sciences, Cumming School of Medicine, University of Calgary, Calgary, AB, Canada.

Received: 25 May 2019 Accepted: 13 August 2019

Published online: 07 October 2019

\section{References}

1. Speich B, von Niederhausern B, Schur N, Hemkens LG, Furst T, Bhatnagar N, et al. Systematic review on costs and resource use of randomized clinical trials shows a lack of transparent and comprehensive data. J Clin Epidemiol. 2018;96:1-11.

2. Gul RB, Ali PA. Clinical trials: the challenge of recruitment and retention of participants. J Clin Nurs. 2010;19(1-2):227-33.

3. Kasenda B, von Elm E, You J, Blumle A, Tomonaga Y, Saccilotto R, et al. Prevalence, characteristics, and publication of discontinued randomized trials. JAMA. 2014;311(10):1045-51.

4. McDonald AM, Knight RC, Campbell MK, Entwistle VA, Grant AM, Cook JA, et al. What influences recruitment to randomised controlled trials? A review of trials funded by two UK funding agencies. Trials. 2006;7:9.

5. Charlson ME, Horwitz RI. Applying results of randomised trials to clinical practice: impact of losses before randomisation. Br Med J (Clin Res Ed). 1984;289(6454):1281-4. 
6. Rendell JM, Licht RW. Under-recruitment of patients for clinical trials: an illustrative example of a failed study. Acta Psychiatr Scand. 2007;115(5):337-9.

7. Nasser N, Grady D, Balke CW. Commentary: improving participant recruitment in clinical and translational research. Acad Med. 2011;86(11):1334-5.

8. Kitterman DR, Cheng SK, Dilts DM, Orwoll ES. The prevalence and economic impact of low-enrolling clinical studies at an academic medical center. Acad Med. 2011;86(11):1360-6.

9. Treweek S, Pitkethly M, Cook J, Fraser C, Mitchell E, Sullivan F, et al. Strategies to improve recruitment to randomised trials. Cochrane Database Syst Rev. 2018;2:Mr000013.

10. Ward E, King M, Lloyd M, Bower P, Friedli K. Conducting randomized trials in general practice: methodological and practical issues. Br J Gen Pract. 1999; 49(448):919-22.

11. Yancey AK, Ortega AN, Kumanyika SK. Effective recruitment and retention of minority research participants. Annu Rev Public Health. 2006;27:1-28.

12. Tramm R, Daws $K$, Schadewaldt V. Clinical trial recruitment-a complex intervention? J Clin Nurs. 2013;22(17-18):2436-43.

13. Witham MD, McMurdo ME. How to get older people included in clinical studies. Drugs Aging. 2007;24(3):187-96.

14. Marcus PM, Lenz S, Sammons D, Black W, Garg K. Recruitment methods employed in the National Lung Screening Trial. J Med Screen. 2012;19(2):94-102.

15. Bjornson-Benson WM, Stibolt TB, Manske KA, Zavela KJ, Youtsey DJ, Buist AS. Monitoring recruitment effectiveness and cost in a clinical trial. Control Clin Trials. 1993;14(2 Suppl):52s-67s.

16. Bracken K, Hague W, Keech A, Conway A, Handelsman DJ, Grossmann M, et al. Recruitment of men to a multi-centre diabetes prevention trial: an evaluation of traditional and online promotional strategies. Trials. 2019;20(1):366

17. Douglas A, Bhopal RS, Bhopal R, Forbes JF, Gill JM, Lawton J, et al. Recruiting South Asians to a lifestyle intervention trial: experiences and lessons from PODOSA (Prevention of Diabetes \& Obesity in South Asians). Trials. 2011;12(1):220.

18. Ranjani H, Weber M, Anjana R, Lakshmi N, Narayan KV, Mohan V. Recruitment challenges in a diabetes prevention trial in a low- and middleincome setting. Diabetes Res Clin Pract. 2015;110(1):51-9.

19. Kusek JW, Ahrens A, Burrows PK, Clarke HS, Foster HE, Hanson K, et al. Recruitment for a clinical trial of drug treatment for benign prostatic hyperplasia. Urology. 2002;59(1):63-7.

20. Kumar N, Crocker T, Smith T, Pow-Sang J, Spiess PE, Egan K, et al. Challenges and potential solutions to meeting accrual goals in a Phase II chemoprevention trial for prostate cancer. Contemp Clin Trials. 2012:33(2):279-85.

21. Bhar SS, Wiltsey-Stirman S, Zembroski D, Mccray L, Oslin DW, Brown GK, et al. Recruiting older men for geriatric suicide research. Int Psychogeriatr. 2012;25(1):88-95.

22. Chlebowski RT, Menon R, Chaisanguanthum RM, Jackson DM. Prospective evaluation of two recruitment strategies for a randomized controlled cancer prevention trial. Clin Trials. 2010;7(6):744-8.

23. Heiney SP, Adams SA, Drake BF, Bryant LH, Bridges L, Hebert JR. Successful subject recruitment for a prostate cancer behavioral intervention trial. Clin Trials. 2010;7(4):411-7.

24. Moinpour CM, Atkinson JO, Thomas SM, Underwood SM, Harvey C, Parzuchowski J, et al. Minority recruitment in the Prostate Cancer Prevention Trial. Ann Epidemiol. 2000;10(8):S85-91.

25. Fletcher JM, Saunders-Smith T, Manns BJ, Tsuyuki R, Hemmelgarn BR, Tonelli M, Campbell DJ. Pharmacist and patient perspectives on recruitment strategies for randomized controlled trials: a qualitative analysis. Trials. In press.

26. Lovato LC, Hill K, Hertert S, Hunninghake DB, Probstfield JL. Recruitment for controlled clinical trials: literature summary and annotated bibliography. Control Clin Trials. 1997;18(4):328-52.

27. UyBico SJ, Pavel S, Gross CP. Recruiting vulnerable populations into research: a systematic review of recruitment interventions. J Gen Intern Med. 2007;22(6):852-63.

28. Huynh L, Johns B, Liu SH, Vedula SS, Li T, Puhan MA. Cost-effectiveness of health research study participant recruitment strategies: a systematic review. Clin Trials. 2014;11(5):576-83.

29. Mody L, Miller DK, McGloin JM, Freeman M, Marcantonio ER, Magaziner J, et al. Recruitment and retention of older adults in aging research. J Am Geriatr Soc. 2008;56(12):2340-8

30. Campbell DJ, Tonelli M, Hemelgarn B, Mitchell C, Tsuyuki R, Ivers N, et al. Assessing outcomes of enhanced chronic disease care through patient education and a value-based formulary study (ACCESS) — study protocol for a 2×2 factorial randomized trial. Implement Sci. 2016;11(1):131.
31. Ghali WA, Knudtson ML. Overview of the Alberta Provincial Project for Outcome Assessment in Coronary Heart Disease. On behalf of the APPROACH investigators. Can J Cardiol. 2000;16(10):1225-30.

32. Usadi RS, Diamond MP, Legro RS, Schlaff WD, Hansen KR, Casson P, et al. Recruitment strategies in two reproductive medicine network infertility trials. Contemp Clin Trials. 2015;45(Pt B):196-200.

33. Krusche A, Rudolf von Rohr I, Muse K, Duggan D, Crane C, Williams JM. An evaluation of the effectiveness of recruitment methods: the staying well after depression randomized controlled trial. Clin Trials. 2014;11(2):141-9.

34. Silagy CA, Campion K, McNeil JJ, Worsam B, Donnan GA, Tonkin AM. Comparison of recruitment strategies for a large-scale clinical trial in the elderly. J Clin Epidemiol. 1991;44(10):1105-14.

35. Borland JM, Aiani LM, Norvelle A, Grantham KN, O'Laughlin K, Terranova J, Fratz KJ, Albers HE. Sex-dependent regulation of social reward by oxytocin receptors in the ventral tegmental area. Neuropsychopharmacology. 2018; 8(44):785-92.

36. Maas AM, Appelman YA. Gender differences in coronary heart disease. Neth Heart J. 2010;18(12):598-602

\section{Publisher's Note}

Springer Nature remains neutral with regard to jurisdictional claims in published maps and institutional affiliations.

\section{Ready to submit your research? Choose BMC and benefit from:}

- fast, convenient online submission

- thorough peer review by experienced researchers in your field

- rapid publication on acceptance

- support for research data, including large and complex data types

- gold Open Access which fosters wider collaboration and increased citations

- maximum visibility for your research: over $100 \mathrm{M}$ website views per year

At $\mathrm{BMC}$, research is always in progress.

Learn more biomedcentral.com/submissions 